\title{
Développement d'outils opérationnels pour la prévision des crues rapides. Prise en compte de la variabilité spatiale de la pluie dans TOPMODEL
}

\author{
The development of tools for flash flood forecasting. \\ The introduction of spatial variability in TOPMODEL.
}

par Rachel Datin*

The aim of this work is to improve the flash flood forecasting (particularly on the Ardèche catchment). There are three main parts. The first one deals with the implementation, in a real time context, of an hydrometeorological forecasting system. The consequences, on discharge simulations, of the uncertainty on average rainfall evaluation (thanks to the raingauge network) are also quantified. The second part of the report concerns the increasing of discharge forecasting terms (until 12 hours, by using a stochastic generator of hourly rainfall scenarios, which was improved in order to integrate other information (qualitative or quantitative) about future rainfalls. At last, we tried to integrate the spatial variability of the rainfall in TOPMODEL : an entirely new version was developed, in order to understand the eventual influence of this spatial variability for the discharge generation.

\section{I INTRODUCTION}

L'objectif principal du travail présenté est de contribuer à l'amélioration de la prévision des crues rapides, sur des bassins versants de quelques centaines de $\mathrm{km}^{2}$, situés dans des régions à relief marqué et soumis à des précipitations parfois extrêmement intenses.

Deux lignes directrices peuvent être distinguées dans cette étude. La première est de répondre à des besoins opérationnels en termes de prévisions des débits, à des échéances comprises entre 6 et 12 heures. Dans ce contexte, nous avons tout d'abord participé à l'élaboration d'une chaîne de prévision des crues, puis développé et amélioré différents outils qui, dès à présent, peuvent être intégrés dans cette chaîne fonctionnant en temps réel (cf. § 2). Dans un deuxième temps, nous avons cherché à préparer de nouveaux éléments qui pourraient soit aider le prévisionniste dans la prise de décision (cf. § 3), soit améliorer la représentation des processus en action sur le bassin, tout en gardant le souci de leur utilisation opérationnelle dans les années qui viennent (cf. § 4).

Le cadre de cette étude a été fourni par le Projet Ardèche, action du Programme Risques Naturels du Plan Etat-Région regroupant Météo France à Lyon, EDF-DTG et le Laboratoire d'étude des Transferts en Hydrologie et Environnement (LTHE) à Grenoble. Le but de ce projet est de tester la faisabilité et la validité d'un outil opérationnel de prévision hydrométéorologique des crues rapides sur le bassin de l'Ardèche. Nous avons travaillé plus spécifiquement sur deux sous-bassins de l'Ardèche : Aulueyres $\left(121 \mathrm{~km}^{2}\right)$ et Vogüé $\left(635 \mathrm{~km}^{2}\right)$ (cf. Figure 1).

Trois parties seront distinguées dans cet article. Les deux premières concernent plus particulièrement les aspects opérationnels de la prévision : au lancement du projet, nous avons d'abord contribué à l'élaboration puis à la mise en œuvre de la chaîne opérationnelle de prévision, en étudiant également l'influence de certaines incertitudes sur la prévision des débits. Nous avons ensuite cherché à étendre les échéances de prévision par l'intégration automatique et objective de prévisions météorologiques dans la chaîne de prévision. Enfin, la troisième et dernière partie s'intéresse à des développements plus cognitifs sur la modélisation pluiedébit, avec, plus particulièrement, la prise en compte de la variabilité spatiale de la pluie.

\footnotetext{
* Rachel Datin a obtenu le prix Henri Milon.
} 


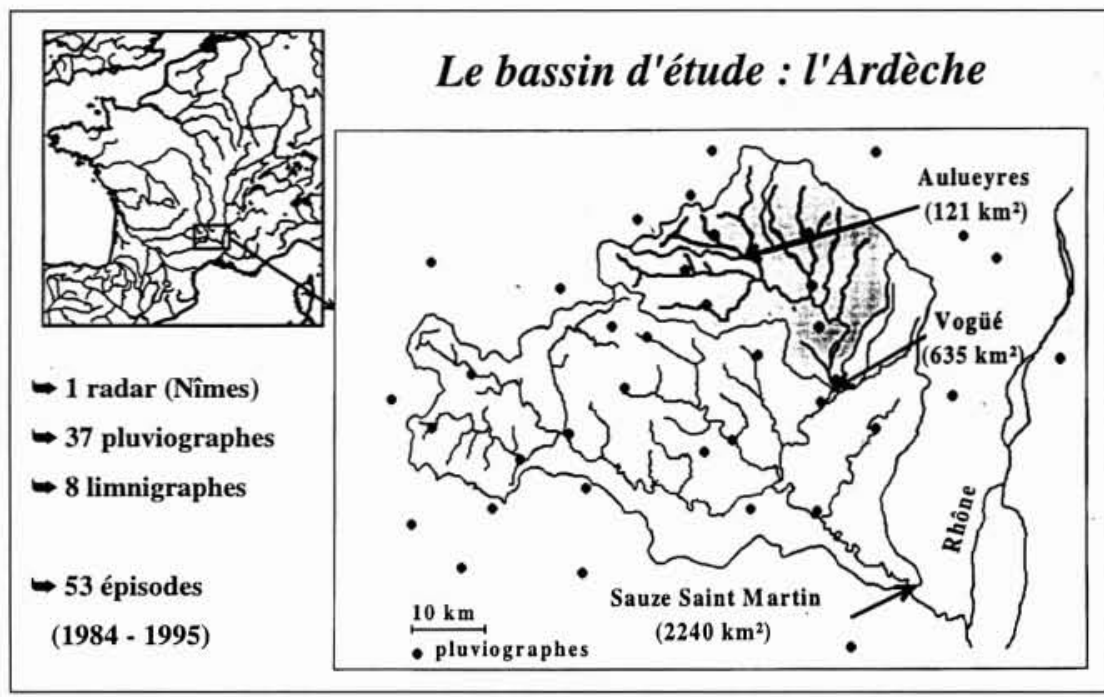

1. Carte du bassin versant de l'Ardèche
DEL " représentera l'ensemble fonction de production et fonction de transfert).

$\mathrm{La}$ version de TOPMODEL que nous avons retenue pour la chaîne opérationnelle fonctionne de manière globale, avec en entrée la pluie moyenne sur le bassin considéré : il nous a donc fallu évaluer, pour chaque pas de temps des 53 épisodes considérés, la lame d'eau moyenne tombée sur chacun des bassins versants. Nous avons pour cela utilisé les deux sources d'information que nous avions à notre disposition : d'une part, les pluies mesurées par les stations pluviométriques (qui, interpolées par la méthode du krigeage permettent d'évaluer des lames d'eau moyennes sur les différents sous-bassins), et d'autre part, les pluies radar, calibrées par Météo

\section{II $\square$ CHAINE OPERATIONNELLE DE PRE- VISIONS DES CRUES. TRAITEMENT DES INCERTITUDES}

L'objectif préliminaire de la première partie a été de constituer une chaîne opérationnelle de prévision hydrométéorologique des crues rapides sur le bassin de l'Ardèche. Après un recensement aussi exhaustif que possible des données disponibles dans cette région, nous avons choisi de travailler sur la période couvrant les années 1984 à 1995. Nous disposions pour celle-ci de 9 stations de mesure de débits, qui définissent donc autant de sous-bassins versants (variant, en superficie, de 19 à $2240 \mathrm{~km}^{2}$ ), et de 37 stations pluviométriques au maximum (le réseau de mesure étant plus dense en 1995 qu'en 1984) pour lesquelles des données horaires sont disponibles (cf. Figure 1). D'autre part, nous disposions également des images horaires du radar de Nîmes depuis 1992. Une sélection d'épisodes pluie-débit nous a permis d'en retenir 53 sur la période considérée, ce qui constitue notre base de données.

Le noyau principal de la chaîne de prévision est constitué d'un modèle hydrologique qui permet de transformer les pluies en débits. Notre choix s'est porté sur TOPMODEL, pour différentes raisons à la fois pratiques et scientifiques : ses hypothèses sont, globalement, bien adaptées aux bassins versants à relief montagneux qui nous intéressent ; d'autre part, des résultats tout à fait satisfaisants avaient été obtenus sur des bassins du même type ; enfin, TOPMODEL est relativement facile à mettre en œuvre. Il est utilisé ici comme une fonction de production, à laquelle nous avons adjoint, pour le transfert du ruissellement à l'exutoire, une fonction de transfert identifiée par la méthode classique de la DPFT, Différence Première de la Fonction de Transfert (dans toute la suite, et dans un souci de simplification, le terme " TOPMO-

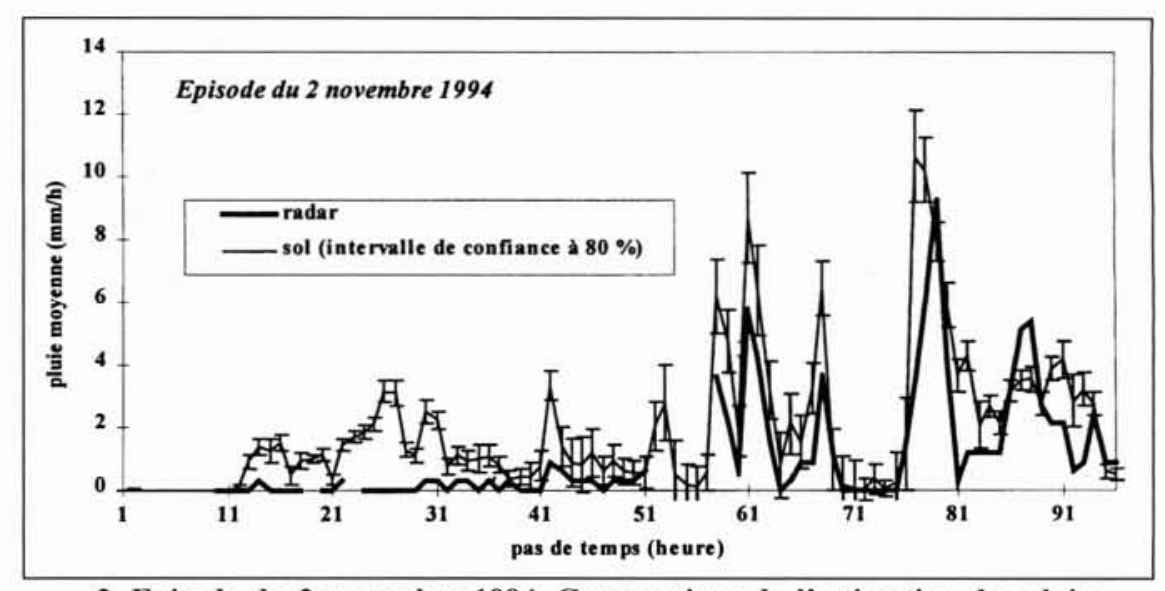

2. Episode du 2 novembre 1994. Comparaison de l'estimation des pluies moyennes sur le bassin versant de Vogüé en utilisant d'une part les stations pluviométriques et d'autre part les données radar. 


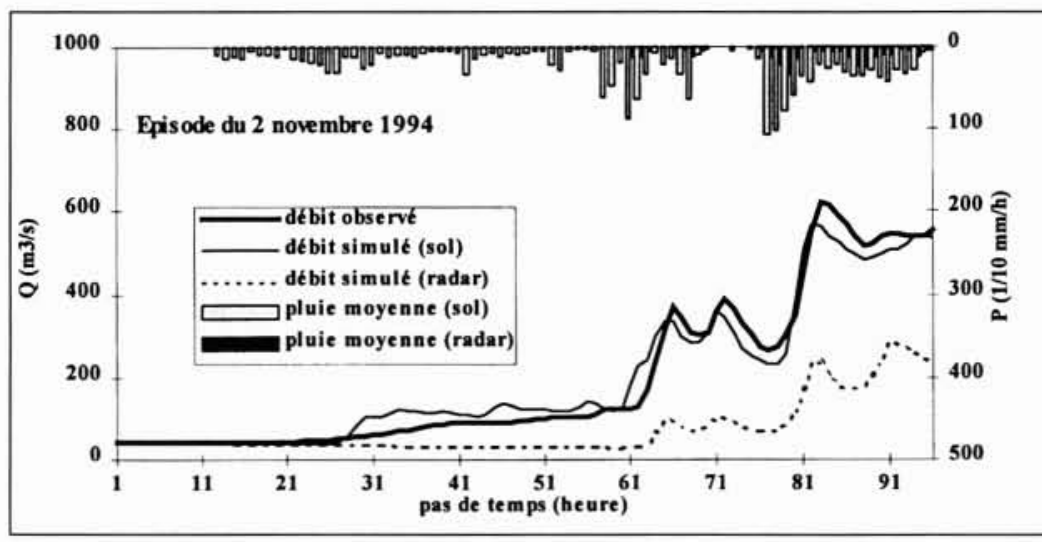

3. Episode du 2 novembre 1994. Comparaison des débits simulés par TOPMODEL en utilisant en entrée les pluies "sol" ou les pluies "radar".

dispose d'un réseau de mesures suffisamment dense (dans notre cas : une station tous les $12 \mathrm{~km}$ environ), l'incertitude sur l'évaluation des lames d'eau moyennes reste faible, et conduit à un suivi des débits tout à fait satisfaisant dans un contexte opérationnel : la figure 4 présente l'épisode du 2 novembre 1994 à Vogüé où les 3 principales stations pluviométriques du bassin sont disponibles. Pour cet épisode, la fourchette d'incertitudes des débits est relativement étroite. En revanche, si le réseau devient trop peu dense (en particulier, en cas de panne ou de foudroiement de certaines stations), l'incertitude sur l'estimation des pluies

elles : l'influence, sur les débits simulés, de l'incertitude sur l'évaluation de la pluie moyenne sur un bassin. En effet, la méthode d'interpolation choisie, le krigeage, fournit, à chaque pas de temps, outre les lames d'eau moyennes, une évaluation de l'incertitude sur ces résultats, par l'intermédiaire d'un écart-type d'estimation.

Nous avons donc bruité les pluies moyennes par des scénarios d'erreur artificiellement générés à partir de cette incertitude théorique. Ceux-ci, fournis en entrée du modèle hydrologique, conduisent à autant de scénarios de débits plus ou moins affectés par ce bruit d'erreur. L'étude des fourchettes de débits ainsi obtenues met en évidence que, si l'on

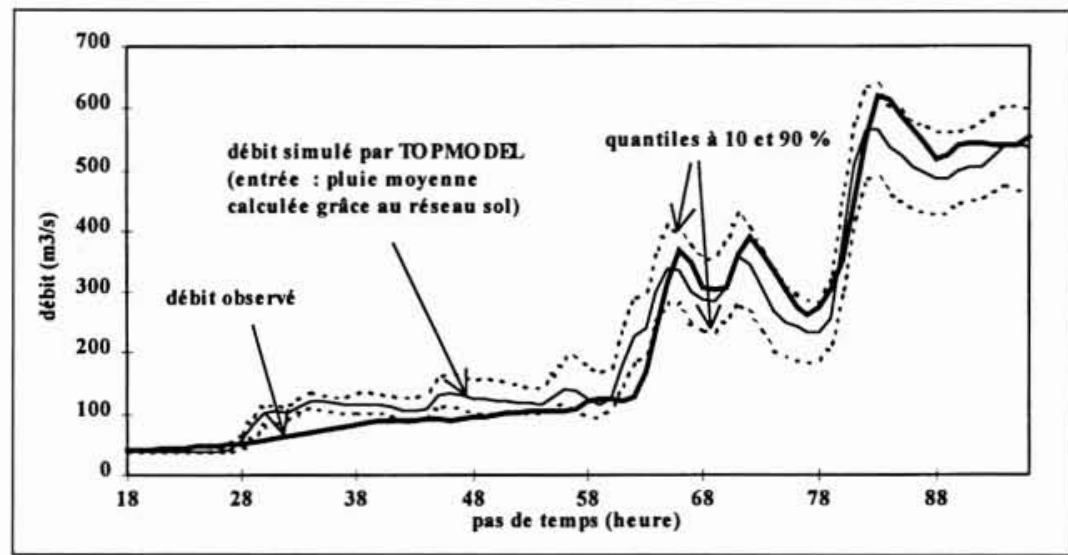

4. Episode du 2 novembre 1994 à Vogüé : évaluation de l'incertitude sur les débits.

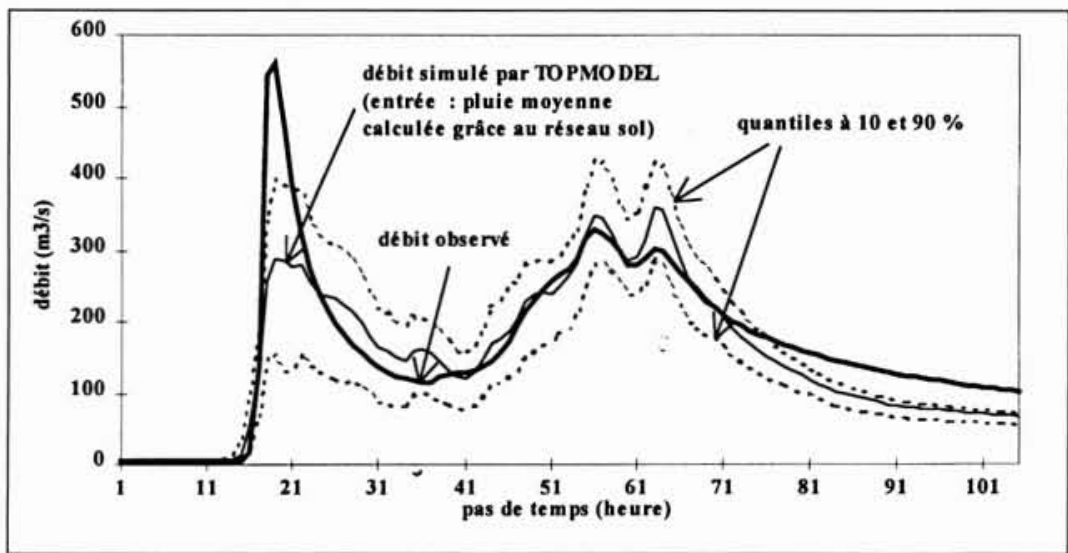

5. Episode du 22 septembre 1993 à Vogié : évaluation de l'incertitude sur les débits. moyennes s'accroît fortement, et rend difficile une estimation correcte des débits. C'est le cas pour l'épisode du 22 septembre 1993 à Vogüé (cf. Figure 5), où une seule des stations pluviométriques principales est disponible : pour cet épisode, la fourchette d'incertitudes est très grande.

Cette étude a donc permis de mettre en évidence l'importance de la densité du réseau de mesures, ou de la bonne localisation d'un radar, pour une évaluation correcte des lames d'eau moyennes, point crucial dans un contexte opérationnel de prévision des débits.

\section{III $\square$ AUGMENTATION DES ÉCHÉANCES DE PRÉ- VISION DES DÉBITS}

A l'issue de cette première partie, essentiellement consacrée à la construction du noyau principal de la chaîne de prévision des crues, il s'est rapidement avéré que, sur les bassins petits ou moyens qui nous intéressent, ce noyau était insuffisant à lui seul pour anticiper les débits suffisamment longtemps à l'avance. En effet, les seules pluies observées jusqu'au pas de temps où l'on souhaite faire la prévision ne permettent d'extrapoler les débits que pour les 2 à 3 heures qui suivent (ce qui correspond approximativement au temps de montée au pic de débit sur les bassins de quelques centaines de $\mathrm{km}^{2}$ qui nous intéressent). Pour une prévision à plus long terme, 6 à 12 heures par exemple (permettant de prendre les mesures nécessaires en cas de forte crue, notamment prévenir les populations éventuellement en péril), il est nécessaire d'étendre les échéances et donc d'utiliser des informations supplémentaires, en particulier sur les pluies futures. Celles-ci peuvent être "moyennes", issues de la climatologie, ou plus spécifiques, grâce à des prévisions de précipitations régulièrement remises à jour . 
Ainsi, pour améliorer l'anticipation des débits, nous avons tout d'abord choisi d'intégrer à la chaîne de prévision un outil permettant de générer des scénarios probables pour les pluies horaires à venir. Celui-ci a fait l'objet de plusieurs études, notamment sur des bassins cévenols : il a donc été repris et adapté à l'un des sous-bassins de l'Ardèche (Aulueyres, $121 \mathrm{~km}^{2}$ ). Les épisodes de pluie générés ont pour contrainte non seulement de respecter la climatologie de la zone d'étude (en termes de durée d'averse, d'intensité maximale par exemple) mais également de respecter le passé immédiat qui vient d'être observé : ainsi, si l'on souhaite imaginer des prolongements

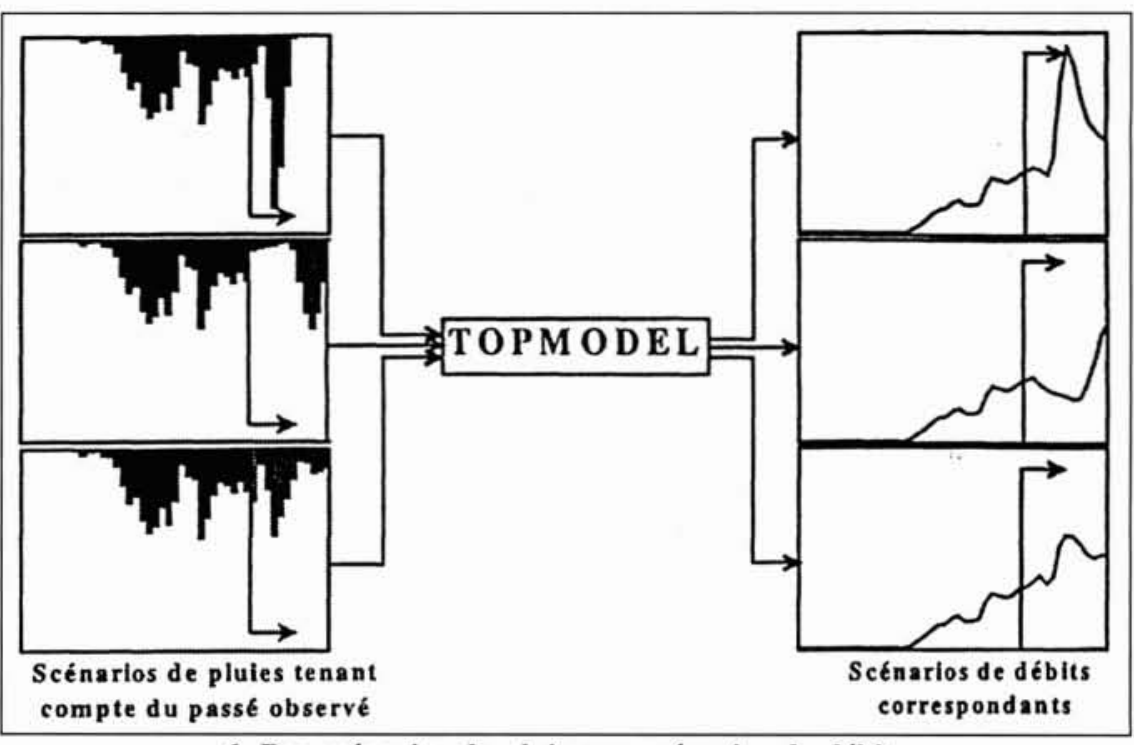

6. Des scénarios de pluie aux scénarios de débits. à l'épisode pluvieux en cours, le générateur doit proposer des suites possibles, acceptables, tenant compte de la pluie déjà survenue. Il est alors possible de générer un grand nombre de scénarios de pluies horaires qui sont ensuite fournis en entrée du modèle hydrologique : il en résulte autant de scénarios de débits probables (cf. Figure 6), dont on peut extraire une prévision, par exemple en considérant les quantiles à 35 et 90\% qui définissent une fourchette indicatrice des débits futurs. Mais à ce stade de l'étude, il est apparu que cette fourchette de débits, essentiellement basée sur la climatologie générale de ces épisodes pluvieux, restait généralement trop large, surtout en début et fin d'épisode, pour permettre une prévision satisfaisante des débits 6 à 12 heures à l'avance et donc améliorer la prise de décision. La Figure 7 présente les résultats obtenus pour l'épisode du 2 novembre 1994 : pour simplifier la lecture des résultats, nous avons choisi de tracer les quantiles à 35 et $90 \%$ des scénarios de débits ; les prévisions obtenues sont comparées à l'un des scénarios utilisés classiquement en prévision opérationnelle, où l'on suppose que la pluie future est nulle.

Nous avons alors cherché à contraindre de manière plus forte les scénarios de pluie en intégrant au générateur des informations sur les pluies futures qui ne soient plus climatologiques, mais spécifiques à l'épisode en cours. Un premier recensement nous a permis de déterminer les informations potentiellement disponibles dans un contexte opérationnel : l'anticipation de l'arrêt des pluies (grâce notamment aux images radar qui permettent de détecter le passage d'un front pluvieux) ; la prévision immédiate des pluies généralement réalisée par advection d'images radar (pour l'heure ou les deux heures à venir); ou encore une prévision quantitative de cumuls journaliers (fournie par des modèles météorologiques ou des méthodes statistiques). Ces informations sont par nature incertaines et fournies, idéalement, sous une forme probabilisée.

Il s'agissait ensuite de réussir à intégrer ces informations dans le générateur pour contraindre les scénarios de pluies horaires. Le cas le plus complexe est de traiter la prévision quantitative des cumuls journaliers. Celle-ci, dont nous disposions de manière opérationnelle, s'appuie sur une tech- nique statistique de recherche, dans le passé, de situations météorologiques analogues à celle du jour considéré. La prévision est alors fournie sous forme d'une distribution probabiliste des pluies pour une journée donnée, conditionnée par la situation météorologique en cours : $\mathrm{x} \%$ de chance de dépasser un cumul $\mathrm{C}$ dans les 24 prochaines heures. A chaque distribution empirique d'analogues est ajustée une loi exponentielle qui permet de reproduire de manière assez satisfaisante le comportement de la distribution empirique (cf. Figure 8).

Les scénarios de pluie générés sont alors contraints à avoir une distribution de cumuls journaliers identiques à celle fournie par la prévision. Mais ce type de prévision est donné, tous les jours, à heure fixe (par exemple, à 8 heures du matin) : si l'on souhaite faire une prévision de débits à une heure quelconque de la journée, la prévision des cumuls journaliers doit alors être régulièrement remise à jour, pour tenir compte de la pluie éventuellement tombée depuis 8 heures du matin. D'autre part, plus la journée avance, et plus l'on souhaite tenir compte également de la prévision du lendemain : il est alors nécessaire de combiner les 2 prévisions (jour $\mathbf{J}$ et $\mathrm{J}+1$ ) des cumuls journaliers pour contraindre plus finement les scénarios de pluies horaires.

Les résultats obtenus par cette approche sur le bassin d'Aulueyres ont démontré l'intérêt d'ajouter des informations supplémentaires sur les pluies futures, et particulièrement celle concernant les cumuls journaliers : les fourchettes de débits prévus se révèlent souvent plus étroites, et plus informatives (cf. Figure 9). Elles permettent notamment une meilleure anticipation de la fin d'un épisode par rapport à l'absence d'information sur le futur. Mais il est également à noter que des informations telles que la prévision immédiate ou l'anticipation de l'arrêt des pluies peuvent également être très pertinentes, surtout si elles viennent compléter une information sur les cumuls des 24 prochaines heures.

L'ultime étape serait maintenant d'intégrer cet outil dans une chaîne de prévision des crues fonctionnant de manière opérationnelle pour tester son apport réel comme aide aux prévisionnistes. 


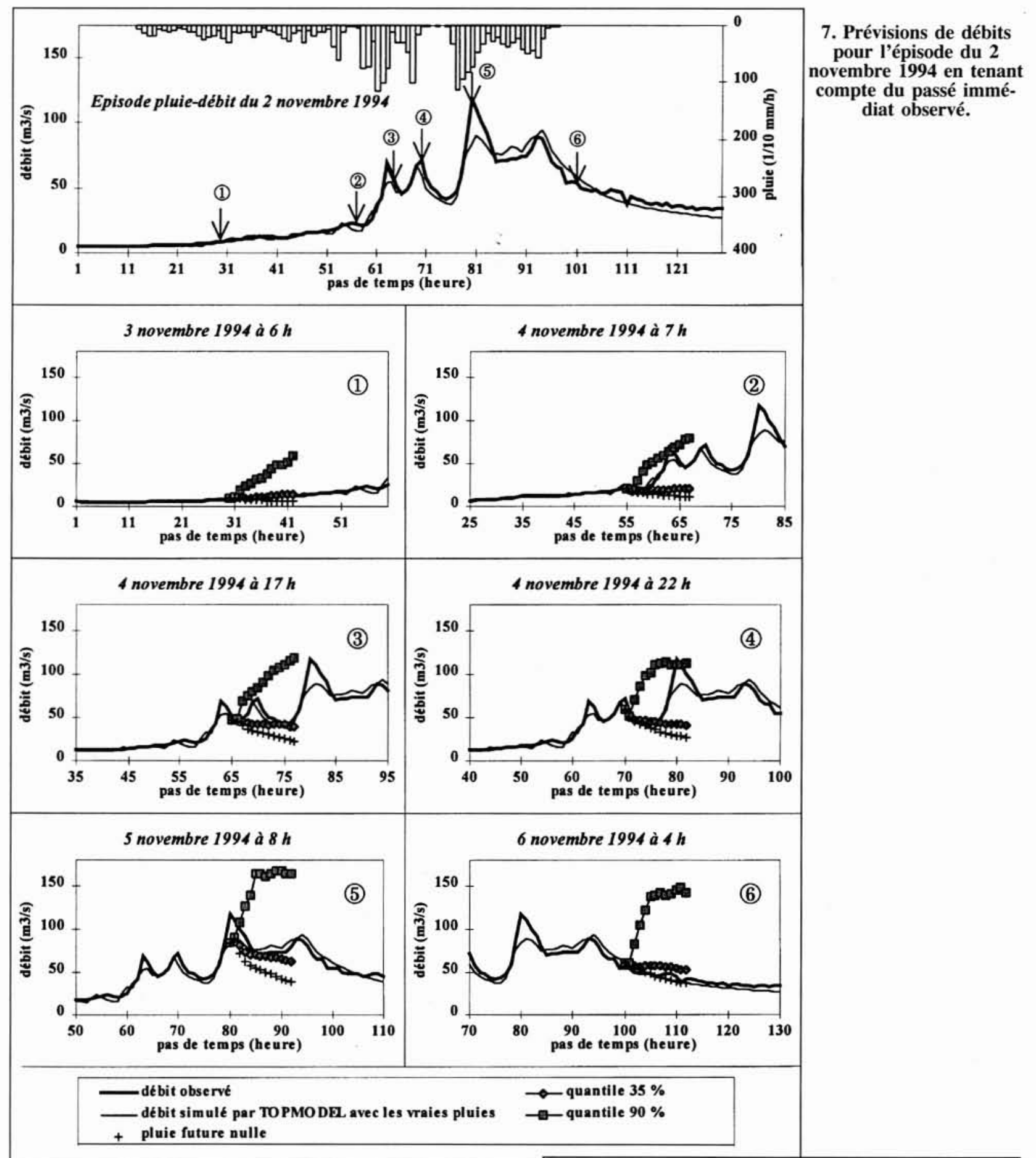

\section{IV $\square$ PRISE EN COMPTE DE LA VARIABI- LITÉ SPATIALE DE LA PLUIE DANS TOPMODEL}

Enfin, après avoir développé une chaîne de prévision des crues pouvant fonctionner en temps réel et donnant des résultats globalement très satisfaisants, nous avons voulu comprendre certaines défaillances du modèle hydrologique. Pour quelques épisodes (en moyenne, un épisode sur 10), celui-ci simulait en effet des débits très différents de ceux réellement observés... C'est le cas en particulier pour l'épisode du 22 septembre 1993 qui est présenté à la Figure 10. L'une des premières raisons qui pouvaient être invoquées est que

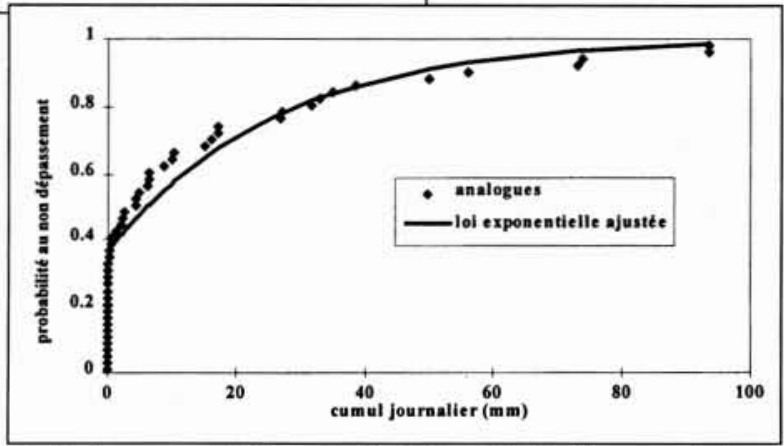

8. Loi exponentielle ajustée à la distribution empirique des analogues obtenues pour la journée du 4 novembre 1993. 


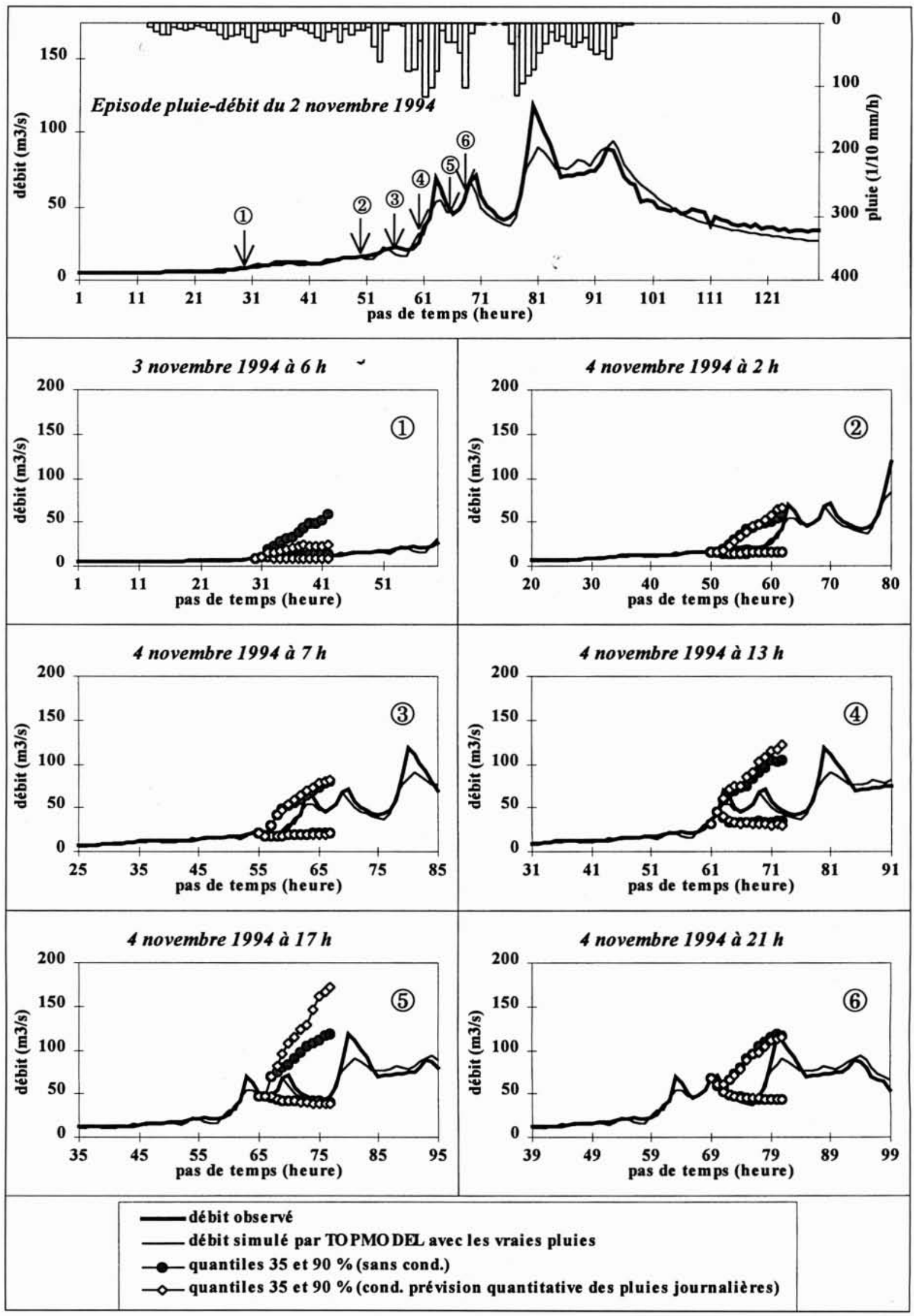

9. Prévisions de débits pour l'épisode du 2 novembre 1994 en tenant compte du passé immédiat observé et d'une prévision des cumuls de pluie pour les 24 prochaines heures. 


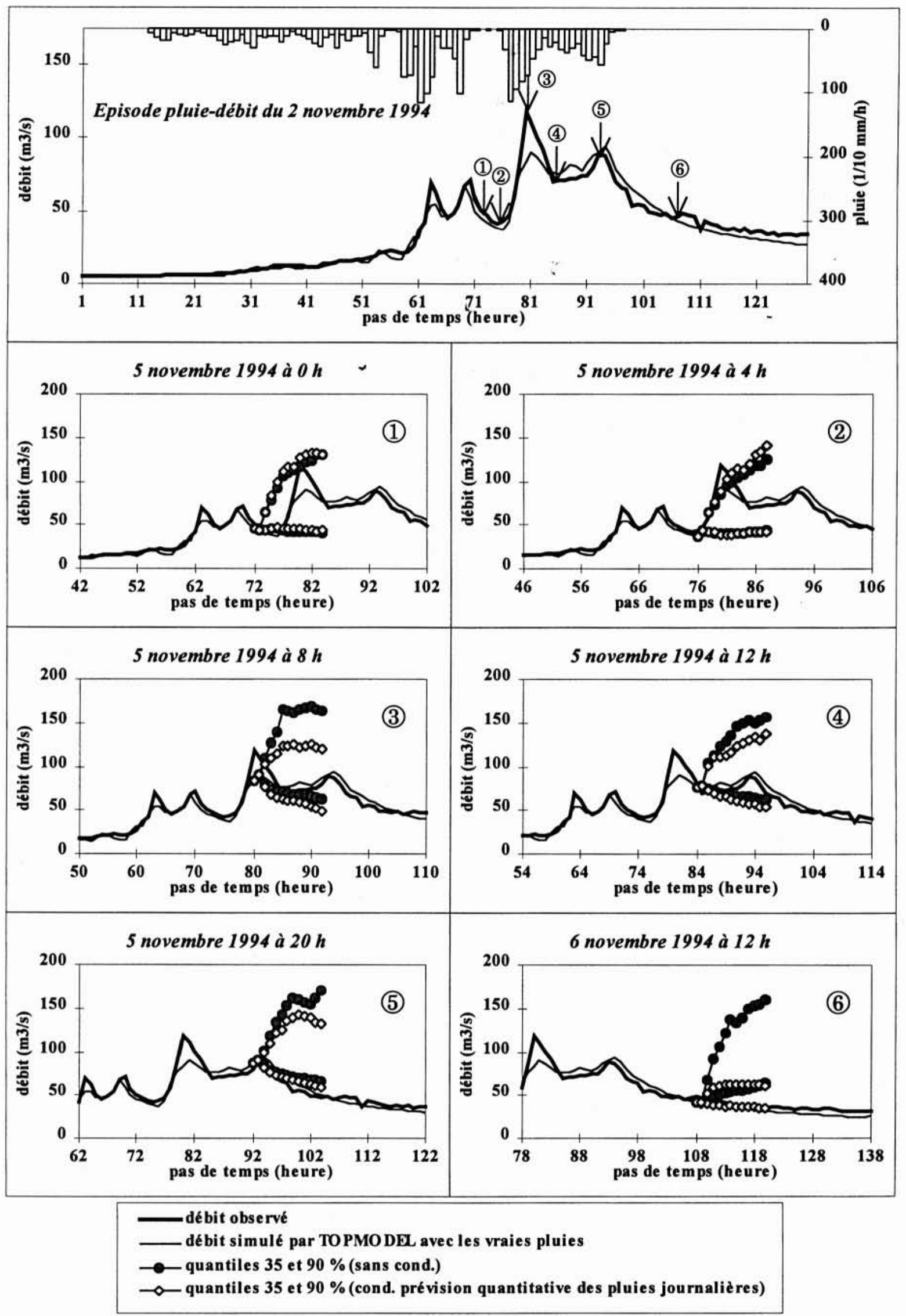

Suite de la figure 9. 
TOPMODEL fonctionne avec une entrée pluie moyenne sur le bassin. Or, sur des bassins versants de quelques centaines de $\mathrm{km} 2$, parfois soumis à des épisodes pluvieux particulièrement violents et souvent très localisés, la variabilité spatiale de la pluie peut être très importante. Pour déterminer si cette variabilité de la pluie pouvait expliquer les "contre-performances" de TOPMODEL pour certains épisodes, nous avons d'abord dû développer une nouvelle version de TOPMODEL, appelée TOPODYN, capable de prendre en compte cette variabilité spatiale de la pluie, tout en conservant les principes de fonctionnement majeurs de la version classique de TOPMODEL. Nous avons, pour ce faire, relaxé deux hypothèses importantes de TOPMODEL : d'une part, l'uniformité spatiale supposée de la recharge des versants, et d'autre part, l'uniformité temporelle supposée des aires drainées.

Ainsi, dans TOPODYN, deux points d'un même bassin versant présentant les mêmes caractéristiques morpho-topographiques (même aire d'alimentation amont et même pente locale) peuvent, dans un même pas de temps, avoir une recharge de versant différente, et ce, notamment en fonction de la localisation de la pluie (ce qui n'était pas le cas dans la version classique de TOPMODEL). Par ailleurs, l'aire drainée qui converge en un point peut évoluer au cours

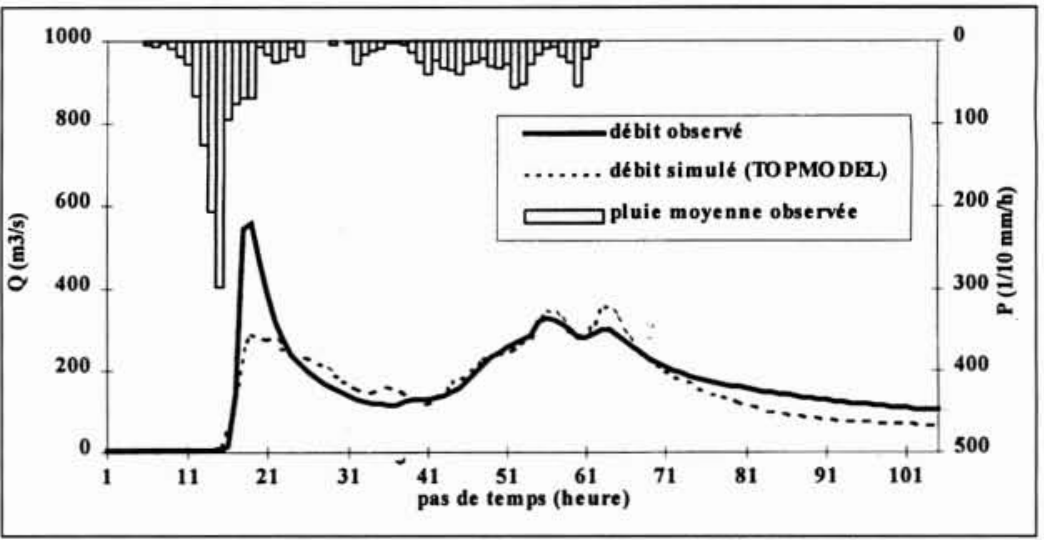

10. Episode du 22 septembre 1993 à Vogüé : modélisation avec TOPMODEL.

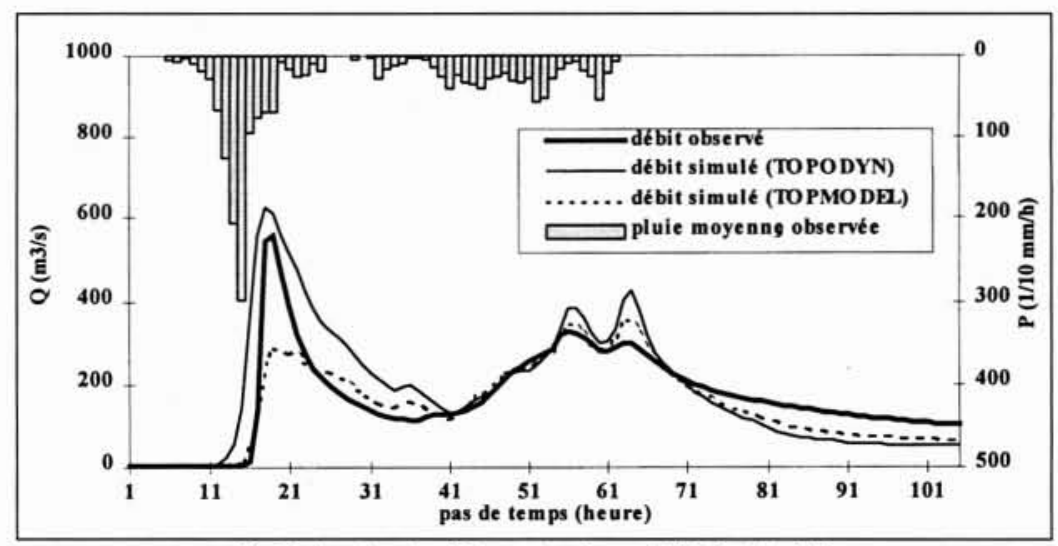

11. Episode du 22 septembre 1993 à Voguié : modélisation avec TOPODYN. d'un épisode pluvieux. Elle n'est donc plus systématiquement égale et constante à l'aire drainée apparente, purement géographique, du point considéré : elle dépend désormais également de la recharge de versant spécifique pour chacun des points situés à son amont.

L'une des conséquences de la relaxation de ces deux hypothèses est que le comportement d'un point du bassin n'est plus seulement conditionné par des données jusqu'alors purement topographiques (aire drainée et pente), mais également par la répartition spatiale de la recharge de versant, variable d'un pas de temps à l'autre, et dépendant, pour partie, de la localisation de la pluie.

Ainsi, l'indice qui, dans TOPMODEL, caractérise un pixel est le suivant :

$$
\lambda_{\mathrm{i}}=\ln \left(\frac{\mathrm{a}_{\mathrm{i}}}{\operatorname{tg} \beta_{\mathrm{i}}}\right)
$$

alors que dans TOPODYN, cet indice, variable dans le temps, intègre la variabilité spatiale de la recharge et celle des aires drainées, et s'exprime de la manière suivante :

$$
\Lambda_{\mathrm{i}, \mathrm{t}}=\ln \left(\frac{\mathrm{a}_{\mathrm{i}, \mathrm{t}} \cdot \mathrm{R}_{\mathrm{i}, \mathrm{t}}}{\operatorname{tg} \beta_{\mathrm{i}}}\right)
$$

La mise en œuvre de TOPODYN sur le bassin de Vogüé $\left(635 \mathrm{~km}^{2}\right)$ avec en entrée du modèle des données de pluies spatialisées, a fourni des résultats tout à fait prometteurs. Dans le cas d'un épisode resté jusqu'alors problématique, l'influence de la variabilité spatiale de la pluie sur la modéli-

sation des débits a pu être clairement démontrée : pour cet épisode, les débits réagissent à la pluie de manière beaucoup plus dynamique et se rapprochent sensiblement des débits réellement observés à l'exutoire du bassin (cf. Figure 11). L'un des faits remarquables de cette nouvelle modélisation est que la sensibilité des débits à la variabilité spatiale de la pluie ne se manifeste pas systématiquement : à ce stade de l'étude, il semble que la morphologie du système pluvieux et, bien sûr, l'intensité des pluies associées, jouent un rôle prépondérant dans la réaction des débits. Des études complémentaires, sur d'autres bassins, et pour d'autres épisodes pluvieux, devront maintenant être menées pour apporter des réponses plus définitives à ces questions, mais l'outil proposé les rend relativement faciles.

\section{$\mathrm{V} \square$ CONCLUSION}

En conclusion, le travail mené dans cette thèse a tout d'abord permis le développement et la mise en œuvre immédiate d'outils d'aide à la prévision des débits pour des bassins versants de quelques centaines de $\mathrm{km}^{2}$ et dans un contexte véritablement opérationnel. Par ailleurs, nous avons proposé d'améliorer l'aide à la décision en prenant en compte objectivement des prévisions de précipitations, et leurs incertitudes intrinsèques. Enfin, l'intégration de la variabilité spatiale de la pluie dans la modélisation pluie-débit a donné des résultats tout à fait prometteurs et confirmé l'intérêt de cette approche, qui sera d'ailleurs poursuivie... 